\title{
Large on/off current ratio in hybrid graphene/BN nanoribbons by transverse electric field-induced control of bandgap
}

Van-Truong Tran, Jérôme Saint-Martin, and Philippe Dollfus

Citation: Appl. Phys. Lett. 105, 073114 (2014); doi: 10.1063/1.4893697

View online: https://doi.org/10.1063/1.4893697

View Table of Contents: http://aip.scitation.org/toc/apl/105/7

Published by the American Institute of Physics

\section{Articles you may be interested in}

Bandgap opening in boron nitride confined armchair graphene nanoribbon

Applied Physics Letters 98, 143107 (2011); 10.1063/1.3571282

Electronic properties of graphene nanoribbons embedded in boron nitride sheets

Applied Physics Letters 95, 123105 (2009); 10.1063/1.3234374

Band gap engineering of graphene/h-BN hybrid superlattices nanoribbons

Journal of Applied Physics 113, 033703 (2013); 10.1063/1.4776208

Suppression of $1 / f$ noise in near-ballistic $h$-BN-graphene-h-BN heterostructure field-effect transistors Applied Physics Letters 107, 023106 (2015); 10.1063/1.4926872

Current-voltage characteristics of a graphene-nanoribbon field-effect transistor Journal of Applied Physics 103, 094510 (2008); 10.1063/1.2917284

Nanoribbons: From fundamentals to state-of-the-art applications Applied Physics Reviews 3, 041302 (2016); 10.1063/1.4966963

\section{LakeShore} CRYOTRONICS

\section{Sensors, Controllers, Monitors}

from the world leader in cryogenic thermometry 


\title{
Large on/off current ratio in hybrid graphene/BN nanoribbons by transverse electric field-induced control of bandgap
}

\author{
Van-Truong Tran, ${ }^{\text {a) }}$ Jérôme Saint-Martin, and Philippe Dollfus ${ }^{\text {b) }}$ \\ Institute of Fundamental Electronics, University of Paris-sud, CNRS, UMR 8622 Orsay, France
}

(Received 12 May 2014; accepted 10 August 2014; published online 20 August 2014)

\begin{abstract}
We propose an approach to design efficient electronic switches based on Graphene/BN heterostructures. By means of atomistic Tight Binding simulations, we investigate heterostructures made of an armchair BN nanoribbon sided by two armchair graphene ribbons where a significant bandgap can be opened. In particular, we show that a bandgap of about $0.55 \mathrm{eV}$ can be strongly suppressed by applying a relatively weak transverse electric field of $10 \mathrm{mV} / \mathrm{A}$. Additionally, by using non-equilibrium Green's function simulation, we show that this effect can be used to control the current in electron devices even at small bias and small length. An on/off current ratio higher than $10^{4}$ is achieved at room temperature. (C) 2014 AIP Publishing LLC.

[http://dx.doi.org/10.1063/1.4893697]
\end{abstract}

Bandgap opening in graphene is a critical issue to make this outstanding material suitable for a wide range of applications. Nanostructuring strategies, as cutting graphene into narrow strips to form graphene nanoribbons $(\mathrm{GNRs})^{1}$ or punching a periodic array of nanoholes into a graphene sheet to form a graphene nanomesh (GNM), ${ }^{2}$ have been shown to be effective to generating a bandgap. However, to achieve a gap of a few hundreds of meV the width of GNRs and neck width of GNMs must be very small, typically smaller than 2-3 nm. Such small dimensions are difficult to achieve and give rise to problems of reproducibility and edge disorder control. ${ }^{3,4}$ It is also possible to use an external electric field to generate and/or modulate the bandgap in graphene or graphene-like structures. This technique has been first demonstrated in carbon nanotubes (CNTs) by placing a CNT in an electric field perpendicular to the tube axis. ${ }^{5}$ In ribbons, a similar effect of transverse electric field applied along the width has been predicted for GNRs, ${ }^{6-8}$ bi-layer GNRs, ${ }^{9}$ and $\mathrm{BN}$ ribbons, ${ }^{10}$ as a consequence of field-induced symmetry breaking. In the case of GNRs, the field may open a small gap in metallic zigzag ribbons. It has a negligible effect on metallic armchair ribbons, ${ }^{6}$ while it reduces the gap of semiconducting ones. However, in an armchair GNR of reasonable width, e.g., with 69 dimer lines, the bandgap reduces only for electric field higher than $4 \mathrm{mV} / \AA$ and can be modulated in a limited range of $0.15 \mathrm{eV}{ }^{6}$ In $\mathrm{BN}$ ribbons, the significant suppression of the large bandgap requires electric fields of strength higher than hundreds or even thousands of $\mathrm{mV} / \AA^{10}{ }^{10}$

An external field applied perpendicularly to the sheets can also open and modulate a bandgap, as shown experimentally in bilayer graphene ${ }^{11}$ and theoretically in bilayer $\mathrm{BN},{ }^{12}$ as a consequence of the difference of potential between both layers. However, using this approach, the controllable bandgap is limited to $0.25 \mathrm{eV}$ in bilayer graphene ${ }^{13}$ and the modulation of bandgap in BN bilayers is not enough to make this material suitable for carrier transport.

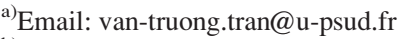

b)Email: philippe.dollfus@u-psud.fr
}

Graphene functionalized with hydrogen $(\mathrm{H})$ or fluorine (F) has been shown to modify strongly the band structure and the transport properties of graphene, with the possibility to open a bandgap that can be adjusted by tuning the $\mathrm{H}$ or $\mathrm{F}$ coverage. ${ }^{14-16}$ The improvement of $I_{\text {on }} / I_{\text {off }}$ ratio in graphene transistors using selective fluorination has been experimentally demonstrated. ${ }^{17}$ Recently, the possibility to grow hybrid monolayers consisting of a mixture of $\mathrm{BN}$ and carbon nanodomains has been demonstrated, ${ }^{18-20}$ with the possibility to form heterostructures, which motivated new strategies of bandgap engineering and proposal of devices. ${ }^{20-22}$ In particular, it has been suggested to introduce a finite-gap hybrid boron-nitride-carbon (BNC) region below the gate of a transistor to improve significantly the $I_{\text {on }} / I_{\text {off }}$ ratio. $^{22}$ A double-BN barrier may be used also to design a resonant tunneling device. ${ }^{22}$

In this Letter, by using an atomistic tight binding (TB) approach, we show that an external transverse electric field applied along the width of an armchair hybrid graphene/BN ribbon can modulate the bandgap over a wide range, typically from more than $0.5 \mathrm{eV}$ to less than $0.08 \mathrm{eV}$. It is worth noting that this effect is obtained for moderate electric fields, smaller than $20 \mathrm{mV} / \AA$. Based on this material study, an electronic device where such a ribbon is connected to two graphene leads is proposed. It should be noted that in this device the transport takes place along the BN/graphene heterostructure, which is expected to provide higher current than for a transport across the heterostructure. According to our ballistic Green's function-based quantum simulations, an on/off current ratio higher than $10^{4}$ can be achieved due to the field-induced modulation of bandgap.

We consider the armchair ribbon heterostructure schematized in Fig. 1, made of a central BN ribbon surrounded by two graphene ribbons. The width of each sub-ribbon is characterized by the number of dimer lines, denoted by $M_{C C 1}, M_{C C 2}$, and $M_{B N}$. For the band structure analysis, the ribbon is considered to be infinite and periodic along $x$ axis. For $M_{C C 1}=M_{C C 2}=15, M_{B N}=29$, i.e., for a total ribbon width $W$ of about $7.1 \mathrm{~nm}$, the calculated bandgap is about $0.55 \mathrm{eV}$. Two side gates are placed along the edges of the ribbon. At these gates, two potentials $V_{1}$ and $V_{2}$ equal to 


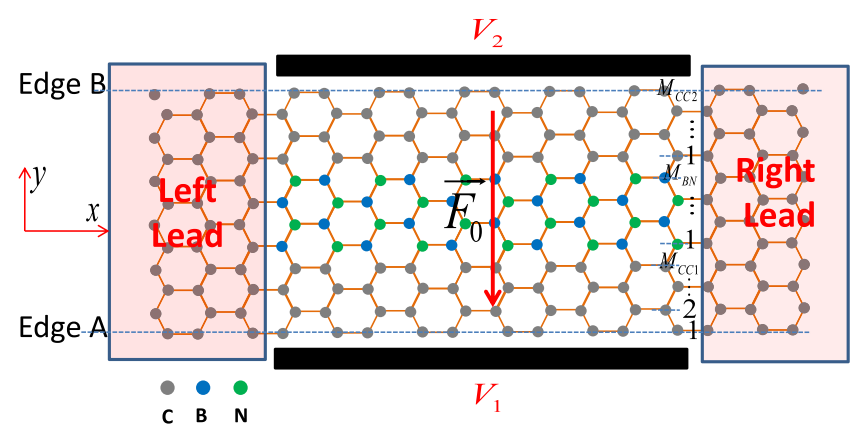

FIG. 1. Schematic view of the Armchair Graphene/BN/Graphene structure studied in this work with $M_{C C 1}=M_{C C 2}=15, M_{B N}=29$. It is placed in between two side gates that generate a transverse electric field. For the transport analysis, a portion of this hybrid ribbon is connected to two graphene contacts.

$-V_{0} / 2$ and $+V_{0} / 2$, respectively, are applied to generate a transverse electric field along the $y$ axis. To make easier the comparison with previous works where the transverse field was assumed to be uniform, we use throughout the letter the concept of average field $F_{0}=V_{0} / W$ which is the strength of the transverse field in free space, i.e., in the absence of Graphene/BN ribbon between the side gates. Though convenient to present the results, this uniform field, called external field, may differ significantly from the actual spacevarying transverse field considered in our self-consistent simulation, as will be shown later.

The electronic energy band structure is investigated using a tight-binding model for the atomic structures limited to the first nearest neighbor interaction, ${ }^{23}$ i.e., using the Hamiltonian

$$
H=\sum_{i} \varepsilon_{i}|i\rangle\left\langle i\left|-\sum_{\langle i, j\rangle} t_{i j}\right| i\right\rangle\left\langle j\left|+\sum_{i} U_{i}\right| i\right\rangle\langle i|
$$

where $\varepsilon_{i}$ is the on-site energy and $U_{i}=-e . V_{i}$ is the electrostatic potential energy at site $i, t_{i j}$ is the hoping parameter between atoms at $i$-th and $j$-th sites. On-site and hoping energies have been taken from Ref. 24 where they have been parameterized to fit first-principles calculation.

In Refs. 6 and 8, the electric field-induced redistribution of charge was neglected and the electrostatic potential in Graphene ribbons was considered as a linear function of atom position along the ribbon width. Here, a self-consistent procedure was performed to compute the potential profile via a coupled 1D Schrödinger-Poisson solver, with Dirichlet boundary conditions of Poisson's equation. Recently, the effect of electric field on the permittivity in graphene has been reported. ${ }^{25}$ It was shown that both in-plane and out-ofplane permittivities may vary significantly under strong field of some hundreds of $m V / \AA$. Moreover, it has been shown that such strong field can also induce mechanical deformation of the lattice that may dramatically change the electronic structure. $^{26,27}$ These phenomena can be neglected in the range of field considered here, i.e., smaller than $20 \mathrm{mV} / \AA$. In the present work, we have assumed the in-plane relative permittivity to be 1.8 in graphene ${ }^{25}$ and 3 in $\mathrm{BN} .{ }^{28}$ The hole and electron densities were computed from the wave functions and eigen-energy values as ${ }^{29}$

$$
\begin{aligned}
& n_{h}(\vec{r})=2 \cdot \sum_{k_{x}} \sum_{\text {valence states }}\left[1-f\left(E_{\mathrm{v}}-E_{N}\right)\right] \cdot\left|\phi_{\mathrm{v}}(\vec{r})\right|^{2} \\
& n_{e}(\vec{r})=2 \cdot \sum_{k_{x}} \sum_{\text {conduction states }} f\left(E_{c}-E_{N}\right) \cdot\left|\phi_{c}(\vec{r})\right|^{2} .
\end{aligned}
$$

From the in-plane charge density $\rho=e\left(n_{h}-n_{e}\right)$, the 3D charge density $\rho_{3 D}=\rho / \Delta z^{30}$ to be introduced into Poisson's equation was obtained by assuming the monolayer thickness $\Delta z$ to be equal to the distance between two graphene layers in graphite, i.e., $\Delta z=0.35 \mathrm{~nm} .{ }^{31}$

In Fig. 2(a), the charge distribution and potential are plotted along $y$ axis for an external electric field $F_{0}$ of $9 \mathrm{mV} / \mathrm{A}$. The charge distribution along the width of the ribbon can be separated in three groups as a function of the atom position along the $y$ direction. The three groups are composed with atoms which have an index position of the form $3 m+1,3 m+2$, and $3 m+3$, respectively, where $m$ is a natural number. This classification of charge is similar to the energy rule demonstrated in armchair graphene nanoribbons. ${ }^{23}$ We checked that it is the same for all other honeycomb armchair structures such as pure graphene and BN ribbons. Fig. 2(a) also shows that under the effect of electric field, free positive and negative charges are separated and the local charge neutrality is no longer ensured. Carriers on electron states tend to move towards the high potential gate and carrier on hole states move towards the low potential gate. Additionally, it is not surprising that the charge density is strongly localized in the graphene regions while the central $\mathrm{BN}$ region is almost free of charges. The dielectric properties of the involved materials reinforce the weakness of the charge density in BN.

In Fig. 2(b), the potential profiles obtained by including (solid line) or not (dashed line) the effect of Charge Redistribution (CR) are plotted for $F_{0}=9 \mathrm{mV} / \AA$. Without CR effect the result can be found analytically by using the continuity conditions of potential and displacement vector at the interfaces whereas the calculation with $\mathrm{CR}$ effect requires a fully numerical self-consistent procedure. As the two potential profiles are different, Fig. 2(b) clearly illustrates that the CR effect is significant for $F_{0}=9 \mathrm{mV} / \AA$. However, at smaller electric field the two potential profiles are very similar (not shown). Actually, the CR effect in this
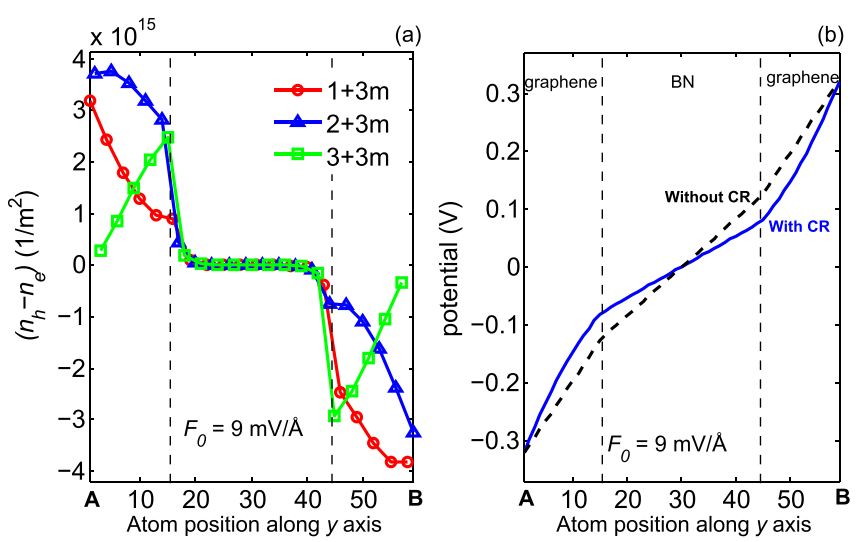

FIG. 2. (a) Redistribution of charge in the system under effect of the electric field. (b) Potential profile along the width of the ribbon with and without effect of CR. Here, the external electric field is $F_{0}=9 \mathrm{mV} / \AA$. 


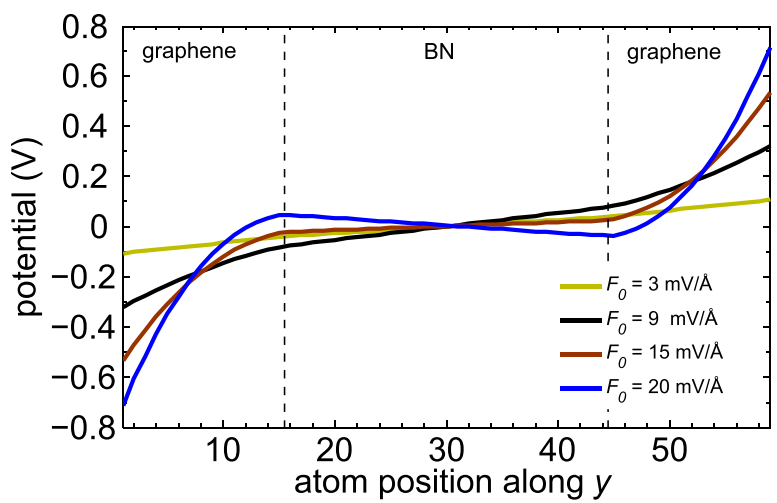

FIG. 3. Typical potential profiles obtained for different external electric fields $F_{0}=3,9,15$, and $20 \mathrm{mV} / \AA$.

structure is negligible for electric fields lower than $6 \mathrm{mV} / \AA$. For larger electric fields, the self-consistent solution is mandatory.

In Fig. 3, the potential profiles (with $\mathrm{CR}$ ) are plotted for different electric fields $F_{O}$ ranging between 3 and $20 \mathrm{mV} / \AA$. At external field high enough to induce significant CR effect, the local electric field in the device is no longer distributed uniformly along the width. While a high field builds up near the ribbon edges where the mobile charges induce a strong screening effect, the field remains relatively small inside the BN sub-ribbon. At external fields $F_{O}$ higher than $15 \mathrm{mV} / \AA$, the direction of the local field in the $\mathrm{BN}$ region even tends to reverse.

The band structure of the studied hetero-structure is displayed in Fig. 4 for different external fields $F_{0}$. The resulting energy gaps are plotted in Fig. 5 as a function of the external field. Both figures show clearly the suppression of energy gap when increasing $F_{O}$. This suppression of bandgap, controlled by the potential applied to the gates, is governed by the effect of the local potential energy $U=-e V$, where $V$ is the local potential which contributes directly to the on-site energy, as expressed in Eq. (1). Thus, at the edge near the gate biased at positive potential, the negative potential energy shifts down the anti-bonding states of the conduction bands, while at the other edge, the positive energy potential shifts up the bonding states of the valence band. This leads to a gradual narrowing of the bandgap when the electric field strength increases. At low external electric field $F_{0}$, these phenomena result in a linear decrease of the bandgap when increasing the potential applied between the gates.

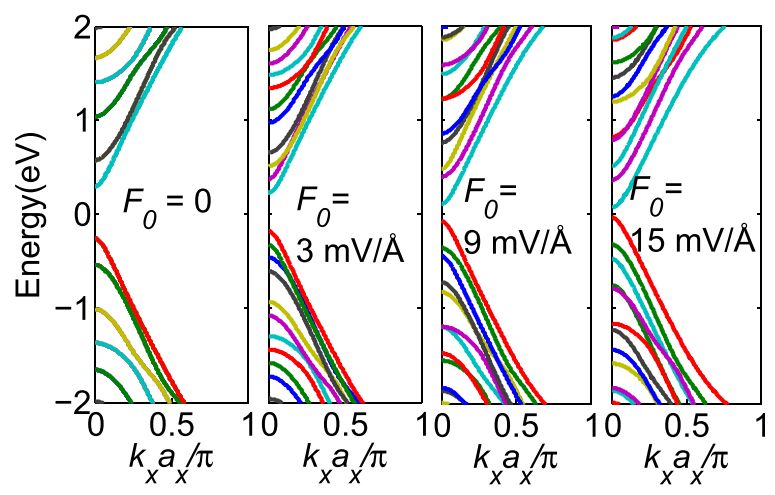

FIG. 4. Plot of energy bands for four values of the external field $F_{0}=0,3,9$, and $15 \mathrm{mV} / \AA$.

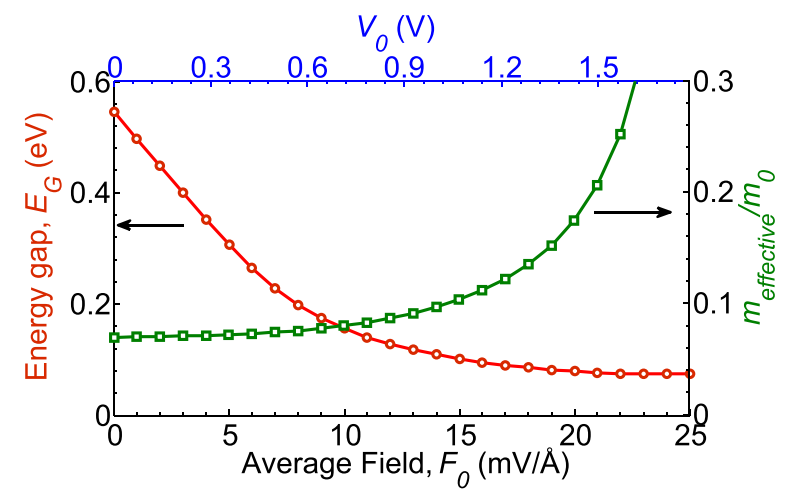

FIG. 5. Energy bandgap (circles) of the hybrid ribbon and effective mass (squares) in the lowest conduction band as a function of the external field $F_{0}$.

Further analysis of Fig. 5 shows that for $F_{0}>6 \mathrm{mV} / \AA$ this linear behavior fails and the gap tends to saturate. When the electric field is larger than $20 \mathrm{mV} / \AA$, the gap reaches the nearly constant value of $74 \mathrm{meV}$. In this structure, the gap cannot be fully suppressed by applying an external field, due to the effect of charge redistribution. At high field, the reduction of the gap is not only related to the strength of the external field but depends also on the strength of the local internal electric field limited by the CR effect as seen in Fig. 2(b) and discussed above.

The electron effective mass in the lowest conduction band was also examined and is plotted in Fig. 5 as a function of the external field. At zero field, the calculated effective mass is relatively small and equal to $0.069 m_{0}$, where $m_{0}$ is the electron rest mass. This effective mass slightly increases up to $0.077 m_{0}$ for a field of $9 \mathrm{mV} / \AA$. At higher field, the effect of charge redistribution affects not only the energy gap but also the shape of the band. The saturation behavior of the gap is associated with a strong increase of the effective mass that reaches $0.174 m_{0}$ at $F_{O}=20 \mathrm{mV} / \AA$. The CR effect limits the band mixing between the lowest conduction band and highest valence band. This resistance to the mixing results in the flattening of the bands.

Electrostatic gates are usually used to control the free charge density in the channel by shifting the Fermi level while the energy gap and the shape of the energy bands remain unchanged. Considering the effect of field-induced bandgap modulation previously highlighted, we propose a way to control the electronic current by using two side gates instead of top and/or back gates. In an electronic switch based on graphene/BN ribbon with two side gates oppositely biased, the induced transverse electric field modulates the energy gap in the active region. Thus the local ambipolar free carrier density and finally the electronic resistance in the active region are controlled. In this field-effect mechanism, at weak electric field the gap should remain large enough to cut off the current. At high electric field the gap tends to vanish and the current is expected to turn on. Following this idea, the device schematized in Fig. 1 has been simulated. The active region is made of a finite portion $(10.5 \mathrm{~nm})$ of the hybrid BN/graphene ribbon studied previously, connected to two graphene leads with a total width of 59 dimer lines. Two gates are located at each side of the active region to generate the transverse electric field. The graphene contacts are 
metallic while the active region is semiconducting at zero transverse field. The simulation was performed at room temperature $(T=300 \mathrm{~K})$ with an initial Fermi energy in the contacts $E_{F}=0.2 \mathrm{eV}$.

The applied voltages in the two side gates of the device are equal to $V_{l}=-E_{F}$ and $V_{2}=V_{O}-E_{F}$, respectively. This bias generates an external field $F_{O}=V_{0} / W$ that is used to modulate the gap in the active region. In the off-regime, i.e., for $F_{O}$ close to zero, as the side gate potential is equal to $-E_{F}$, the Fermi level in the contacts is aligned to the middle of the large gap formed in the active region. Hence, a very low offcurrent is expected. The increase of the potential $V_{0}$, applied on side gate 2, turns on the device. Indeed, the associated increase of $F_{0}$ induces a reduction of the band-gap that finally increases the value of the current. Moreover, the increase of the potential $V_{0}$ generates a standard field-effect, i.e., it induces an increase of the charge density in the device which reinforces the effect of the bandgap suppression to switch on the device. Thus, an excellent on-off current ratio is expected in this device.

The simulation of electron transport was performed within the ballistic approximation. The quantum transport was treated by means of the previous tight-binding description coupled to the non-equilibrium Green's function approach $^{29,32}$ where the transmission $T(E)$ is computed as $T=\operatorname{Trace}\left(\Gamma_{L} G \Gamma_{R} G^{\dagger}\right)$, where $G=\left[E-H_{D}-\Sigma_{L}-\Sigma_{R}\right]^{-1}$ is the retarded Green's function of the device, and $\Gamma_{L(R)}$ $=i\left(\Sigma_{L(R)}-\Sigma_{L(R)}^{\dagger}\right)$ is the injection rate at the left (right) contact characterized by the self-energy $\Sigma_{L(R)}$. Finally, the current was computed from the transmission using the Landauer's formula. ${ }^{29}$

In Fig. 6, the current is plotted as a function of the bias voltage $V_{\text {bias }}$ applied between the graphene leads for transverse electric fields $F_{0}=0,5,10$ and $20 \mathrm{mV} / \AA$. As the energy bandgap is gradually suppressed, it is remarkable that the current increases strongly. The red curve with circles, that corresponds to $F_{0}=0 \mathrm{mV} / \AA$, exhibits very weak current at low bias because the chemical potentials in the leads do not cross available energy states in the active region. Nevertheless, due to the finite and small size of the active region, some evanescent waves can tunnel through the active region. Once the transverse electric field is high enough, the gap is strongly suppressed and the current is widely enhanced due to the

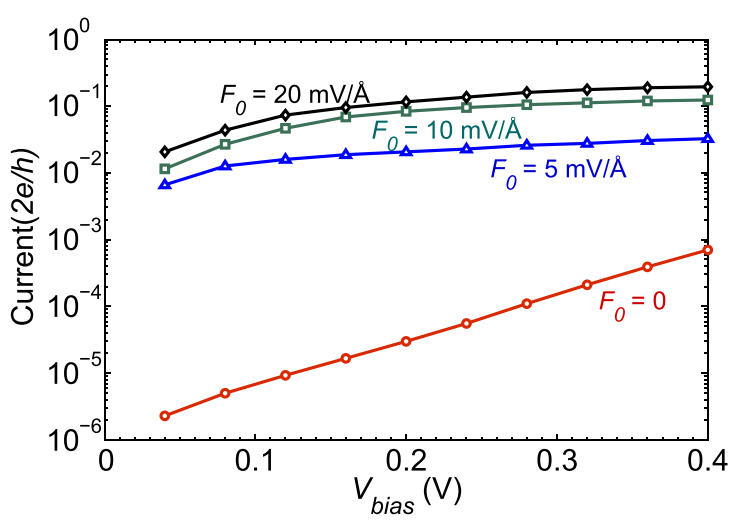

FIG. 6. Current as a function of bias voltage for transverse electric fields $F_{0}=0,5,10$ and $20 \mathrm{mV} / \AA$. The simulation was performed at room temperature $T=300 \mathrm{~K}$, and for $E_{F}=0.2 \mathrm{eV}$ in the leads. increasing contribution of propagating waves. At low $F_{0}$, the rapid increase of current when $F_{0}$ increases is consistent with the bandgap reduction observed in Fig. 5. For fields higher than about $10-15 \mathrm{mV} / \AA$, the bandgap tends to saturate, which leads to a limited increase of current. Actually, two effects contribute to limit the current at high transverse field: the quasi-saturation of bandgap and the increase of effective mass, as shown in Fig. 5. For this particular ribbon, at $V_{\text {bias }}=0.2 \mathrm{~V}$ the on/off current ratio $I_{O N} / I_{O F F}$ defined as the ratio of currents at $F_{O}=20 \mathrm{mV} / \AA$ and at $F_{O}=0 \mathrm{mV} / \AA$ reaches the nice value of 3830 , and even 14200 if the length of the active region increases to $17 \mathrm{~nm}$ (not shown), due to the lower transparency of the active region.

In conclusion, we have demonstrated the effect of a moderate transverse electric field on the effective modulation and suppression of the energy bandgap in an armchair Graphene/BN structure, by considering carefully the effect of charge redistribution at high field. We have shown that the gap can be modulated from $0.55 \mathrm{eV}$ down to values lower than $0.1 \mathrm{eV}$ by applying a transverse electric field of about $15 \mathrm{mV} / \AA$. Beyond this value of field, the reduction of bandgap becomes limited, as a consequence of the effect of charge redistribution, and the effective mass increases strongly, which makes the field range $0-15 \mathrm{mV} / \AA$ the most relevant to control the band structure of the ribbon studied here. Taking advantage of this effect, we have proposed an efficient method for controlling the current in devices by using side gates conveniently biased. This could open new approaches to controlling electron current in small graphene and graphene-like devices.

We would like to thank V. Hung Nguyen for fruitful discussions. This work was partially supported by the Agence Nationale de la Recherche (ANR) through project NOE (ANR 12 JS03 006 01).

${ }^{1}$ M. Han, B. Özyilmaz, Y. Zhang, and P. Kim, Phys. Rev. Lett. 98, 206805 (2007).

${ }^{2}$ J. Bai, X. Zhong, S. Jiang, Y. Huang, and X. Duan, Nat. Nanotechnol. 5, 190 (2010).

${ }^{3}$ V. Hung Nguyen, M. Chung Nguyen, H. Viet Nguyen, and P. Dollfus, J. Appl. Phys. 113, 013702 (2013).

${ }^{4}$ D. Querlioz, Y. Apertet, A. Valentin, K. Huet, A. Bournel, S. GaldinRetailleau, and P. Dollfus, Appl. Phys. Lett. 92, 042108 (2008).

${ }^{5}$ J. O'Keeffe, C. Wei, and K. Cho, Appl. Phys. Lett. 80, 676 (2002).

${ }^{6}$ C. P. Chang, Y. C. Huang, C. L. Lu, J. H. Ho, T. S. Li, and M. F. Lin, Carbon 44, 508 (2006).

${ }^{7}$ Y. Son, M. Cohen, and S. Louie, Nature 444, 347 (2006).

${ }^{8}$ H. Chung, M. Lee, C. Chang, Y. Huang, and M. Lin, J. Phys. Soc. Jpn. 80, 044602 (2011).

${ }^{9}$ H. Chung, P. Yang, T. Li, and M. Lin, Philos. Mag. 94, 1859 (2014).

${ }^{10}$ Z. Zhang and W. Guo, Phys. Rev. B 77, 075403 (2008).

${ }^{11}$ J. B. Oostinga, H. B. Heersche, X. Liu, A. F. Morpurgo, and L. M. K. Vandersypen, Nat. Mater. 7, 151 (2008).

${ }^{12}$ Z. Yang and J. Ni, J. Appl. Phys. 107, 104301 (2010).

${ }^{13}$ F. Xia, D. B. Farmer, Y.-M. Lin, and P. Avouris, Nano Lett. 10, 715 (2010).

${ }^{14}$ D. Elias, R. Nair, and T. Mohiuddin, Science 323, 610 (2009).

${ }^{15}$ R. R. Nair, W. Ren, R. Jalil, I. Riaz, V. G. Kravets, L. Britnell, P. Blake, F. Schedin, A. S. Mayorov, S. Yuan, M. I. Katsnelson, H.-M. Cheng, W. Strupinski, L. G. Bulusheva, A. V. Okotrub, I. V. Grigorieva, A. N. Grigorenko, K. S. Novoselov, and A. K. Geim, Small 6, 2877 (2010).

${ }^{16}$ S. Bruzzone and G. Fiori, Appl. Phys. Lett. 99, 222108 (2011).

${ }^{17}$ K. I. Ho, J. H. Liao, C. H. Huang, C. L. Hsu, W. Zhang, A. Y. Lu, L. J. Li, C. S. Lai, and C. Y. Su, Small 10, 989 (2014). 
${ }^{18}$ L. Ci, L. Song, C. Jin, D. Jariwala, D. Wu, Y. Li, A. Srivastava, Z. F. Wang, K. Storr, L. Balicas, F. Liu, and P. M. Ajayan, Nat. Mater. 9, 430 (2010).

${ }^{19}$ P. Sutter, R. Cortes, J. Lahiri, and E. Sutter, Nano Lett. 12, 4869 (2012).

${ }^{20}$ Z. Liu, L. Ma, G. Shi, W. Zhou, Y. Gong, S. Lei, X. Yang, J. Zhang, J. Yu, K. P. Hackenberg, A. Babakhani, J.-C. Idrobo, R. Vajtai, J. Lou, and P. M. Ajayan Nat. Nanotechnol. 8, 119 (2013).

${ }^{21}$ V. Hung Nguyen, F. Mazzamuto, A. Bournel, and P. Dollfus, J. Phys. D: Appl. Phys. 45, 325104 (2012).

${ }^{22}$ G. Fiori, A. Betti, S. Bruzzone, and G. Iannaccone, ACS Nano 6, 2642 (2012).

${ }^{23}$ H. Zheng, Z. Wang, T. Luo, Q. Shi, and J. Chen, Phys. Rev. B 75, 165414 (2007).

${ }^{24}$ G. Seol and J. Guo, Appl. Phys. Lett. 98, 143107 (2011).
${ }^{25}$ E. J. G. Santos and E. Kaxiras, Nano Lett. 13, 898 (2013).

${ }^{26}$ W. Guo and Y. Guo, Phys. Rev. Lett. 91, 115501 (2003).

${ }^{27}$ C. Tang, W. Guo, and Y. Guo, Appl. Phys. Lett. 88, 243112 (2006).

${ }^{28}$ C. R. Dean, A. F. Young, I. Meric, C. Lee, L. Wang, S. Sorgenfrei, K. Watanabe, T. Taniguchi, P. Kim, K. L. Shepard, and J. Hone, Nat. Nanotechnol. 5, 722 (2010).

${ }^{29}$ S. Datta, Quantum Transport: Atom to Transistor (Cambridge University Press, Cambridge, 2005).

${ }^{30}$ T. Andrijauskas, Lith. J. Phys. 52, 63 (2012).

${ }^{31}$ A. Hashimoto, K. Suenaga, K. Urita, T. Shimada, T. Sugai, S. Bandow, H. Shinohara, and S. Iijima, Phys. Rev. Lett. 94, 045504 (2005).

${ }^{32}$ C. H. Lewenkopf and E. R. Mucciolo, J. Comput. Electron. 12, 203 (2013). 